\title{
INFLUENCE OF TECHNICAL CONDITION OF CONTROL AND MEASUREMENT EQUIPMENT ON CALIBRATION RESULTS
}

doi: 10.2478/czoto-2021-0009

Date of submission of the article to the Editor: 06/12/2020

Date of acceptance of the article by the Editor: 25/03/2021

Małgorzata Kawalec ${ }^{1}$ - orcid id: 0000-0003-3612-0971

Czerwińska Karolina ${ }^{1}$ - orcid id: 0000-0003-2150-0963

Andrzej Pacana ${ }^{1}$ - orcid id: 0000-0003-1121-6352

${ }^{1}$ Rzeszow University of Technology, Poland

\begin{abstract}
In the paper there are analyzed the procedures used in the process of calibrating concerning control and measurement equipment. There was assumed the mathematical model of the measuring instrument which was then analyzed. The factors that result from the imperfect technical condition of the control and measurement equipment and may have an impact on the measurement results were also analyzed. Models of errors' models were assumed that may affect the calibration results. The static calibration of control and measurement equipment was analyzed. The results of numerical simulations concerning the static calibration of control and measurement equipment were presented, taking into account the linear and non-linear processing functions of the measurement equipment. The results of computations obtained for various models of processing functions were compared with each other and conclusions were drawn regarding the accuracy of the mapping of the measured quantity.
\end{abstract}

Keywords: management engineering, measurement equipment, static calibration, processing functions, numerical simulations, regression analysis

\section{INTRODUCTION}

Contemporary enterprises using measuring instruments must adapt to the specific conditions and guidelines set by quality management systems. An example would be a standard (ISO 9001:2015a) which states that that reliable measurement results can only be obtained if the measuring equipment is, inter alia:

- calibrated at specified intervals or before use against units of measurement associated with national or international measurement units standards,

- adjustable,

- protected against damage that may occur during transport,

- protected during storage, etc.

An important element of ensuring the quality of production processes is proper supervision over the control and measurement equipment. In metrological terminology, 
there are terms related to the activities verifying the correct operation of control and measurement instruments, such as: calibration, control, measurement traceability, reference standard, checking, non-compliance, defect, adjustment, specification, measurement laboratory. Each of these terms has a different use:

- Calibration - according to (ISO/IEC Guide 99:2010) is an action which - under certain conditions - in the first step determines the relationship between the values of the quantity mapped by the measurement standard (along with their measurement uncertainties) and the corresponding indications (along with their uncertainties), and in the second step uses this information to determine the relationship that allows to obtain the result based on the indication. In turn, according to (ISO 14978:2018) (pkt. 3.8) calibration is a set of operations establishing, under certain conditions, the relationship between the values of the measured quantity indicated by the measuring instrument or measuring system - or the values represented by the standard of measurement or reference material, and the corresponding values of the quantity realized by the standard of the measurement unit. To explain this, the calibration may be limited to determining errors of indications or corrections of indications of the instrument at selected points of the measuring range.

- Inspection - its purpose is to determine compliance between one or more features of a product or service and the established requirements.

- Traceability - is a property of a measurement result or a standard for a measurement unit where the result may be related most often to a standard of measurement, using an unbroken chain of calibrations, all of which contribute to the uncertainty of measurement (ISO/IEC Guide 99:2010).

- Nonconformity - is a failure to meet the requirement (International Dictionary of Basic and General Metrology Terms, 1993; ISO 9000:2015a).

- Reference standard - is the unit of measure standard.

- Verification - according to standard includes activities allowing to determine the compliance of the measuring instrument with the requirements of standards, international recommendations and technical conditions (ISO 9000:2015a; ISO 9001:2015b).

- Adjustment - is a set of activities performed at the measurement system, ensuring that the appropriate indications correspond to the values of the quantity (IEC 60050$301: 2000)$.

It should be especially emphasized that for the efficient functioning of measuring instruments, it is extremely important to regularly calibrate or check them. Laboratories performing these activities must have precise procedures so that calibration and checking are carried out according to strictly defined methods, in accordance with national and international standards, as well as with the recommendations of international organizations. These procedures must be legible and known to laboratory personnel and contain specific information, such as: name of the instrument to be calibrated, parameters to be specified, equipment used to perform the calibration, reference standards, environmental conditions, uncertainty estimation procedure, protocol of measurement results (Arendarski et al., 2009; Jakubiec and Malinowski, 2004; Jakubiec et al., 2014; Siwiec and Pacana, 2021).

Many factors affect the correctness of calibrations and checking measuring instruments carried out in laboratories. These are, for example: methods used, premises conditions, equipment, reference of measurements to standards of measurement units, 
qualifications of staff, environmental conditions, etc. (Ostasz et al., 2020; Pacana et al., 2018). The following may have a negative impact: location of the laboratory in the vicinity of vibration sources, electromagnetic influence, a nearby heat source, sunlight shining on instruments, inadequate room lighting, temperature, air humidity (Siwiec et al., 2019). For the correct performance of the process of calibration and checking of measuring instruments, a strict procedure should be established, including the reference standards used. This procedure should ensure compliance of calibration and measurements with the SI standards of measurement units. The measurement laboratory should also have a procedure for calibrating and checking its reference standards. These standards should be calibrated by an entity that can ensure traceability. Calibration or verification of a measuring instrument by a given laboratory is confirmed by issuing a verification certificate, which should include a declaration of compliance or non-compliance with the specified conditions. According to the standard PN-EN ISO/IEC 17025 the calibration certificate should contain the results and uncertainties of the measurements (Arendarski et al., 2009; ISO/IEC 17025:2017, Jakubiec and Malinowski, 2004).

\section{METHODOLOGY OF RESEARCH}

In order to present a mathematical model of a measuring instrument, one must give input quantities and an output quantity. Control and measurement instruments can be characterized by design parameters and parameters, the change of which reflects the adjustment of the instrument. The causal relationship between the input and output of an instrument may be expressed as a relationship:

$$
y=g\left(x_{1}, x_{2}, \ldots, x_{n-1}, x_{n}, a_{1}, a_{2}, \ldots, a_{k}, b_{1}, b_{2}, \ldots, b_{l}\right)
$$

where: $a_{1}, a_{2}, \ldots, a_{k}-$ design parameters, e.g., element dimensions, relations representing constraints, etc., $b_{1}, b_{2}, \ldots, b_{1}$-adjustable parameters that are subject to a deliberate change, e.g. when changing the measuring range of a multi-range instrument, when setting the set position of the measuring value, etc. Since the design and regulated parameters must remain constant during the measurement, in the further analysis of the processing function model, one can adopt a processing function model that is simpler than the formula (1), in the form of:

$$
y=f\left(x_{1}, x_{2}, \ldots, x_{n-1}, x_{n}\right)
$$

where: $y$ - output quantity, $x_{1}, x_{2}, \ldots, x_{n}$ - input quantities, function $f\left(x_{1}, x_{2}, \ldots, x_{n}\right)$ is the processing function of the instrument and equation (1) is the processing equation of the instrument with which the measurement is performed.

Among the input quantities, one of them is measured, e.g. $x_{n}$, and the remaining $x_{1}, x_{2}$, $\ldots, x_{n-1}$ are quantities that affect the resulting quantity $y$. If the measurements are carried out in accordance with a discrete measurement scheme, each of the quantities $x_{\mathrm{i}}$, where $i=1,2, \ldots, n$ is represented by the vector $X_{i}$, and the output quantity $y$ is represented by the vector $Y$. During the measurement, the control and measurement instrument is also influenced by disturbing quantities. Some of these values are immeasurable and therefore have to be omitted from the model.

Calibration of the control and measurement device is aimed at giving the output value $y$ of the device a measure of the input value $x$, i.e. determining the function $[5,19,22]$ :

$$
\varphi(y)=x^{*}
$$


where: $\varphi(y)$ - function mapping the calibration procedure, $y$ - the output size of the instrument, $x^{*}$ - measure of the measured quantity, determined on the basis of the instrument indications. In general, the measured quantity $x$ and its measure, indicated by the instrument, are different, so there is an error which is calculated as the following difference:

$$
\delta=x^{*}-x
$$

where: $\delta$-instrument error, $x^{*}$ - measure of the measured quantity, determined on the basis of the instrument indications, $x$ - measured input quantity.

The instrument calibration procedure should lead to the fact that the above-mentioned the instrument error is 0 . However, in general the task is not easy, since the processing function $f(1)$ depends on many parameters. Thus, the solution of the inverse problem, leading to the determination of the inverse function $f^{1}$, is difficult. In order for the calibration problem to be possible, it is carried out under conditions in which it can be ensured that the influencing quantities $x_{1}, x_{2}, \ldots, x_{n-1}$ have the values determined during the calibration. Therefore, in the calibration conditions, the processing function $f_{0}(x)$ can be used, which depends only on one input quantity, which is the measured quantity, i.e.:

$$
y_{0}=f_{o}\left(c_{1}, c_{2}, \ldots, c_{n-1}, x\right)=f_{0}(x)
$$

where: $c_{i}=$ const, $i=1,2, \ldots, n-1$ - constants in the course of the quantity calibration procedure, corresponding $c_{i}=$ const, $i=1,2, \ldots, n-1, x=x_{n}$ - measured quantity, $f_{0}(x)-$ instrument processing function in the calibration procedure.

Usually, the function representing the calibration procedure is written as a polynomial:

$$
y_{0}=f_{0}(x)=p_{o}+p_{1} x+p_{2} x^{2}+\ldots+p_{k} x^{k}
$$

The results of measurements carried out with a given measuring instrument are affected by a number of measurement errors. In the modeling used in the described studies, it was assumed that the resulting error can be expressed by the formula:

$$
\delta_{\text {res }}=\delta_{\text {sys }}+\delta_{\text {rand }}+\delta_{b}+\delta_{\text {ad }}
$$

where:

- the resulting error $\delta_{\text {res }}$ - error which is the resultant of the following component errors;

- systematic error $\delta_{\text {sys }}$ - error which, after multiple measurements of the same state of the quantity measured with the same instrument, under the same conditions has a constant value or changes according to a known dependence;

- random error $\delta_{\text {rand }}$ - error which changes randomly under the same conditions when the same state of a quantity measured with the same instrument is repeatedly measured;

- basic error $\delta_{b}$ - error in reference conditions associated with the assumed influence quantity values $x_{i}$;

- additional errors $\delta_{a d}$ - errors caused by the different conditions of the measurement execution from the reference conditions.

The desired form of the processing function for many control and measurement instruments is a linear function. If it is not a linear function, the tendency is usually to linearize the processing function. Linearization, i.e. approximation of the processing characteristics of the instrument, given in the form of discrete information $\left(x_{\mathrm{i}}, y_{\mathrm{i}}\right), i=1,2$, $\ldots, n$ as a function of the first degree. In the case of control and measurement devices with a clear non-linear characteristic, it should be approximated by the best-matched function. Due to the most common mild variability of the nonlinear characteristic of the 
measuring instrument, this approximation is performed using the mean square criterion in the form:

$$
R=\sum_{i=0}^{n}\left[z\left(x_{i}\right)-f\left(x_{i}\right)\right]^{2} \rightarrow \min
$$

where: $R$-cumulative deviation, remainder, $x_{i}$-abscissas of measurement points, $f(x)$ - approximated measuring instrument processing function, $z(x)$ - an approximating function of a measuring instrument processing function defined in discrete form, $n+1-$ the number of measurements made when determining the characteristics of a measuring instrument. The criterion (8) leads to the least squares method (Draper and Smith, 1981; Lawson and Hanson, 1974). The formulas used in modeling the processing function of a measuring instrument in the process of its calibration are derived below. Due to the fact that in the approximation of the processing function of the measuring instrument not only the polynomial function can be used, it was assumed that the vector of the base approximation functions has the general form:

$$
\Phi(x)=\left\{\phi_{0}(x), \phi_{1}(x), \ldots, \phi_{n}(x)\right\}=\left[\phi_{0}(x), \phi_{1}(x), \ldots, \phi_{n}(x)\right]^{T}
$$

where: $\Phi(x)$ - vector of basis functions, $\varphi_{i}(x)$ - $i$-th basis function, [ai $]$ - vector row containing elements $a_{i},[]^{\top}-$ row vector transposition, $\left[a_{i}\right]^{\top}=\left\{a^{i}\right\},\left\{a^{i}\right\}$ - vector column containing elements $a_{\mathrm{i}}$. The vector of the coefficients of the approximating function was defined as:

$$
P=\left[p_{0}, p_{1}, \ldots, p_{k}\right]
$$

where: $P$ - row Victor containing coefficients $p_{\mathrm{i}}, i=1,2, \ldots, k$. The vector of the coefficients of the approximating function was defined as:

$$
\begin{gathered}
z(x)=p_{0} \varphi_{o}(x)+p_{1} \varphi_{1}(x)+\ldots+p_{k} \varphi_{k}(x) \\
z(x)=P \cdot \Phi(x)
\end{gathered}
$$

where the multiplication on the right side of formula (12) means the dot product.

Minimization of the residual function $R$ :

$$
R\left(p_{0}, p_{1}, \ldots, p_{k}\right)=\sum_{i=0}^{n}\left[p_{0} \varphi_{0}\left(x_{i}\right)+p_{1} \varphi_{1}\left(x_{i}\right)+\ldots+p_{k} \varphi_{k}\left(x_{i}\right)-z\left(x_{i}\right)\right]^{2}
$$

requires the computation of first partial derivatives:

$$
\frac{\partial R}{\partial p_{j}}\left(p_{0}, p_{1}, \ldots, p_{k}\right)=2 \sum_{i=0}^{n}\left[p_{0} \varphi_{0}\left(x_{i}\right)+p_{1} \varphi_{1}\left(x_{i}\right)+\ldots+p_{k} \varphi_{k}\left(x_{i}\right)-z\left(x_{i}\right)\right] \varphi_{j}\left(x_{i}\right)
$$

and equating them to zero, which leads to the following system of $k+1$ linear equations with $k+1$ unknowns:

$$
\begin{gathered}
p_{0} \sum_{i=0}^{n} \varphi_{0}^{2}\left(x_{i}\right)+p_{1} \sum_{i=0}^{n} \varphi_{0}\left(x_{i}\right) \varphi_{1}\left(x_{i}\right)+\ldots+p_{k} \sum_{i=0}^{n} \varphi_{0}\left(x_{i}\right) \varphi_{k}\left(x_{i}\right)=\sum_{i=0}^{n} \varphi_{0}\left(x_{i}\right) z\left(x_{i}\right) \\
\ldots \\
p_{0} \sum_{i=0}^{n} \varphi_{0}\left(x_{i}\right) \varphi_{k}\left(x_{i}\right)+p_{1} \sum_{i=0}^{n} \varphi_{0}\left(x_{i}\right) \varphi_{k}\left(x_{i}\right)+\ldots+p_{k} \sum_{i=0}^{n} \varphi_{k}^{2}\left(x_{i}\right)=\sum_{i=0}^{n} \varphi_{k}\left(x_{i}\right) z\left(x_{i}\right)
\end{gathered}
$$

Assuming the input matrix and the ordinate vector of the form: 


$$
U=\left[\begin{array}{cccc}
\varphi_{0}\left(x_{1}\right) & \varphi_{1}\left(x_{1}\right) & \ldots & \varphi_{k}\left(x_{1}\right) \\
\varphi_{0}\left(x_{2}\right) & \varphi_{1}\left(x_{2}\right) & \ldots & \varphi_{k}\left(x_{2}\right) \\
\ldots & \ldots & \ldots & \ldots \\
\varphi_{0}\left(x_{n}\right) & \varphi_{1}\left(x_{n}\right) & \ldots & \varphi_{k}\left(x_{n}\right)
\end{array}\right], \quad Z=\left[\begin{array}{c}
z\left(x_{1}\right) \\
z\left(x_{2}\right) \\
\ldots \\
z\left(x_{n}\right)
\end{array}\right]
$$

we will obtain a general solution to the problem of approximation used in the research:

$$
U^{T} U P=U^{T} Z
$$

If the main matrix $U^{\top} U$ of the system (17) is non-singular the coefficients of the approximating function can be unequivocally calculated using the following formula:

$$
P=\left(U^{T} U\right)^{-1} U^{T} Z \text { for } \operatorname{det}\left(U^{T} U\right) \neq 0
$$

In the simulations, apart from the matrix determinant, also the condition number of the main $U^{\top} U$ matrix of the system of equations (17) was calculated. The reason is the high sensitivity of the solution results to errors in the $Z$ vector, defined in (16), which may occur in measurements with the use of various measuring devices (Doyle, 2004). The errors $\delta Z$ in vector $Z$ cause errors $\delta P$ in the vector of coefficients $P$ which can be written as the following formula:

$$
\left(U^{T} U\right)(P+\delta P)=Z+\delta Z
$$

Taking into account equations (17) - (19) and using the Cauchy-Schwartz inequality concerning the norm of the matrix product (Bellman, 1960; Horn and Johnson, 1986) it can be written that:

$$
\left\|U^{T} U\right\|\|P\| \geq\|Z\| \quad \text { and } \quad\left\|\left(U^{T} U\right)^{-1}\right\|\|\delta Z\| \geq\|\delta P\|
$$

where $\|\mathrm{A}\|$ denotes the Euclidean norm of a matrix $A$. Therefore, the following inequality can be obtained:

$$
\frac{\|\delta P\|}{\|P\|} \leq \frac{\left\|\left(U^{T} U\right)^{-1}\right\|\|\delta Z\|}{\|P\|}
$$

The right side of the inequality (21) can be limited by the following upper bound:

$$
\frac{\left\|\left(U^{T} U\right)^{-1}\right\|\|\delta Z\|}{\|P\|} \leq \frac{\left\|\left(U^{T} U\right)^{-1}\right\|\left\|U^{T} U\right\|\|\delta Z\|}{\|Z\|}
$$

That leads to inequality:

$$
\frac{\|\delta P\|}{\|P\|} \leq \frac{\left\|\left(U^{T} U\right)^{-1}\right\|\left\|U^{T} U\right\|\|\delta Z\|}{\|Z\|}
$$

Taking into account that the condition number of the matrix $\mathrm{A}$ is given by the formula:

$$
\operatorname{cond}(A)=\left\|A^{-1}\right\|\|A\|
$$

on the basis of formula (22) and inequality (23) it can be written that

$$
\frac{\|\delta P\|}{\|P\|} \leq \operatorname{cond}\left(U^{T} U\right) \frac{\|\delta Z\|}{\|Z\|}
$$

The condition number of the real and symmetric matrix can be calculated from the ratio of the eigenvalues of the matrix (Wilkinson, 1965): 


$$
\operatorname{cond}\left(U^{T} U\right)=\frac{\mid \lambda_{\max }\left[U^{T} U\right]}{\mid \lambda_{\min }\left[U^{T} U\right]}
$$

where: $\lambda_{\max }\left(U^{\top} U\right)$ and $\lambda_{\min }\left(U^{\top} U\right)$ - the maximum eigenvalue and the minimum eigenvalue of the symmetric $U^{\top} U$ matrix, respectively. From the inequality (25) it can be concluded that the condition number of the $U^{\top} U$ matrix plays the role of a multiplier that can significantly increase the upper limit of relative error which originates in the procedure of determining the coefficients of the function approximating considered instrument processing function. These errors induced by the condition of the measuring instrument and the errors which occur in during measurements made for its calibration. In the case of bad condition number of the main matrix $U^{\top} U$ of the system of equations (17), i.e. when it is too large number, the setup of experiment, e.g. the setup of measuring points might be changed in order to reduce the condition number. Another way is the application of numerical methods and algorithms developed for solving illconditioned system of equations including regularization of matrices obtained during computations (Tikhonov and Arsenin, 1977).

\section{RESULTS AND DISCUSSION}

In the case of the linear model of the measuring instrument processing function, it was assumed that the ideal function is given by the formula:

$$
y(x)=a x+b
$$

50 points were generated, evenly distributed over the range $\langle 0,50.0\rangle$. representing measurements in the instrument calibration procedure. The measurements are distorted by the presence of the resulting error with a normal distribution:

$$
y(x)=a x+b+N(A, \sigma)
$$

where: $A$ - noise mean, $\sigma$ - noise standard deviation, $N(A, \sigma)$ - Gaussian noise function. The function approximating the measuring instrument processing function was searched for in the form:

$$
y^{*}(x)=a^{*} x+b^{*}
$$

The results for the linear model are in tab. 1 and for $N(0.0,2.0), N(1.0,2.0)$ in Fig. 2.

Table 1

Results of computations for a linear model (28) of the instrument processing function $f(x)$ for model coefficients $a=0.5$ and $b=1.0$. Computed values of $\operatorname{cond}\left(U^{\top} U\right)<3280$ in all cases.

\begin{tabular}{|l|l|l|l|l|}
\hline Noise mean A & $\begin{array}{c}\text { Noise standard } \\
\text { deviation } \boldsymbol{\sigma}\end{array}$ & $\begin{array}{c}\text { Residual Sum of } \\
\text { Squares RSS }\end{array}$ & $\begin{array}{c}\text { Computed } \\
\text { estimator } \mathbf{a}^{*}\end{array}$ & $\begin{array}{c}\text { Computed } \\
\text { estimator } \mathbf{b}^{*}\end{array}$ \\
\hline 0.0 & 1.3 & 11.01 & 0.486 & 1.770 \\
\hline 1.0 & 1.3 & 50.45 & 0.496 & 2.093 \\
\hline 0.0 & 2.0 & 19.39 & 0.478 & 1.029 \\
\hline 1.0 & 119.0 & 0.509 & 2.308 \\
\hline
\end{tabular}

The nonlinear model of the instrument processing function, taking into account the presence of normal noise $N(A, \sigma)$, had the form:

$$
y(x)=a x^{3}+b x^{2}+c x+d+N(A, \sigma)
$$



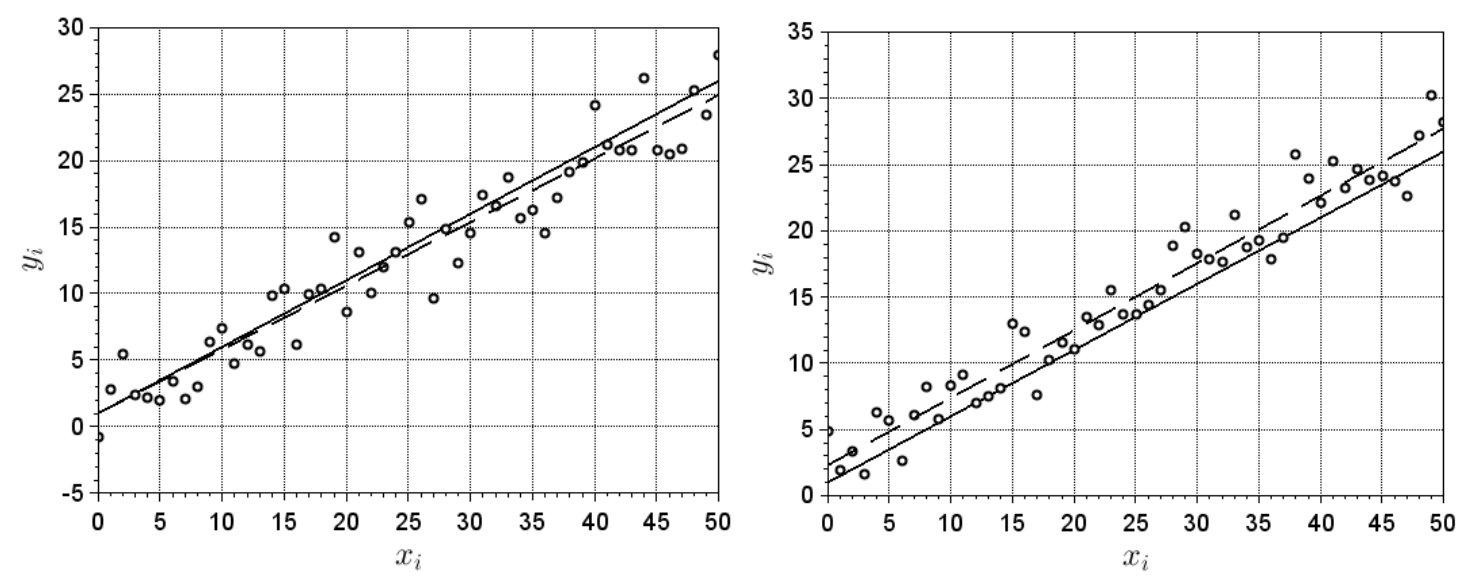

Fig. 1. Examples of linear models: on the left - for normal noise $N(A, \sigma)=N(0.0,2.0)$ and to the right for normal noise $N(A, \sigma)=N(1.0,2.0)$;

continuous line - ideal model, dashed line - approximated model

The calculations for the model (30) were carried out for 57 measurement points evenly distributed in the interval $\langle 0,34.0\rangle$. The calculation results for the nonlinear model are given in tab. 2 and shown for $N(0.0,2.0)$ and $N(1.0,2.0)$ in Fig. 2.

Table 2

Results of computations for a nonlinear model (30) of the instrument processing function $f(x)$ for model coefficients $a=-0.01, b=0.5, c=-0.7, d=0.5$. Computed values of $\operatorname{cond}\left(U^{\top} U\right) \approx$ $3.18 \mathrm{e} 9$ in all cases.

\begin{tabular}{|l|l|l|l|l|l|l|}
\hline $\begin{array}{c}\text { Noise } \\
\text { mean A }\end{array}$ & $\begin{array}{c}\text { Noise } \\
\text { standard } \\
\text { deviation } \mathbf{\sigma}\end{array}$ & $\begin{array}{c}\text { Residual } \\
\text { Sum of } \\
\text { Squares } \\
\text { RSS }\end{array}$ & $\begin{array}{c}\text { Computed } \\
\text { estimator } \\
\mathbf{a}^{*}\end{array}$ & $\begin{array}{c}\text { Computed } \\
\text { estimator } \\
\mathbf{b}^{*}\end{array}$ & $\begin{array}{c}\text { Computed } \\
\text { estimator } \\
\mathbf{c}^{*}\end{array}$ & $\begin{array}{c}\text { Computed } \\
\text { estimator } \\
\mathbf{d}^{*}\end{array}$ \\
\hline 0.0 & 1.3 & 7.76 & 4.898 & -0.574 & 0.493 & -0.01 \\
\hline 1.0 & 1.3 & 76.39 & 6.103 & -0.631 & 0.493 & -0.01 \\
\hline 0.0 & 2.0 & 5.83 & 4.977 & -0.846 & 0.512 & -0.01 \\
\hline 1.0 & 2.0 & 97.44 & 7.530 & -0.887 & 0.507 & -0.01 \\
\hline 0.0 & 14.0 & 543.81 & 0.395 & -0.816 & 0.544 & -0.01 \\
\hline 7.0 & 14.0 & 2598.96 & 16.430 & -1.362 & 0.508 & -0.01 \\
\hline
\end{tabular}
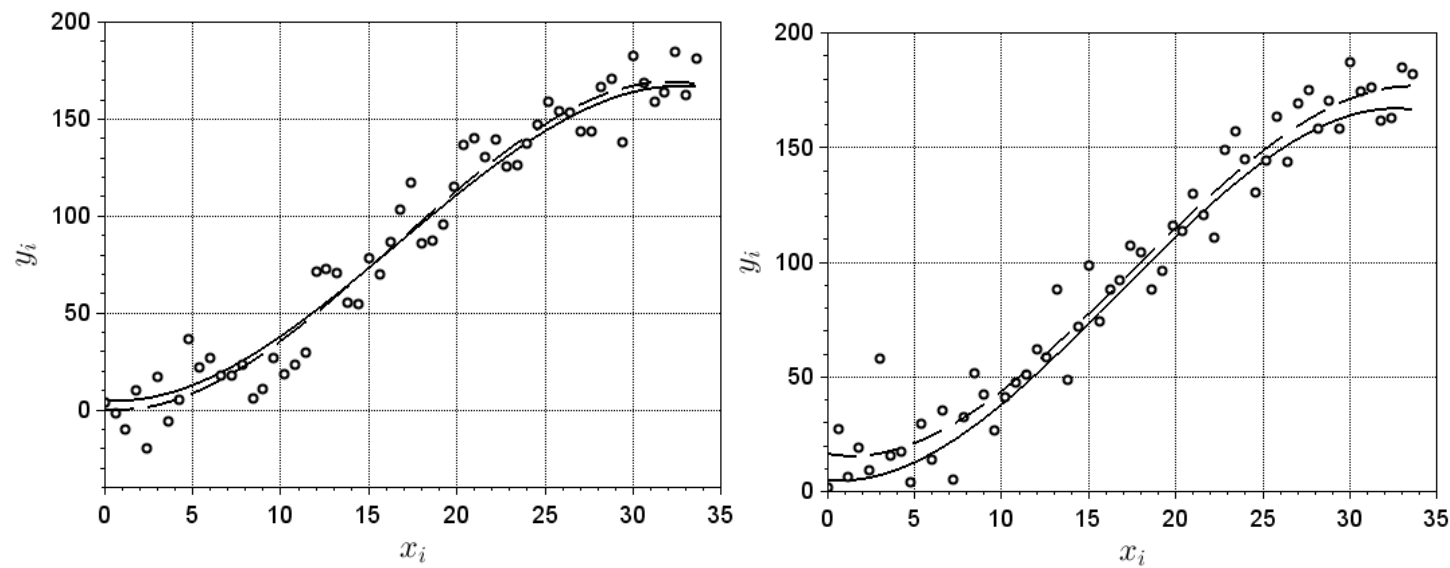

Fig. 2. Examples of nonlinear models: on the left - for normal noise $N(A, \sigma)=N(0.0,14.0)$ and to the right for normal noise $N(A, \sigma)=N(7.0,14.0)$; continuous line - ideal model, dashed line - approximated model 
The nonlinear model was loaded with noise with a normal distribution and parameters the same as the linear model of the processing function. The simulation was also performed for a noise level much higher than in the linear model $N(A, \sigma)=N(0.0,14.0)$ and $N(A, \sigma)=N(0.0,14.0)$ in order to maintain a comparable signal mean value.

The analysis of the results presented in tab. 1 and 2 indicates that the linear model is characterized by a much lower value of the $U^{\top} U$ matrix conditioning index than the nonlinear model. The adoption of the mean value of Gaussian noise, representing measurement errors, had a significant impact on the value of the residual sum of squares RSS. The coefficient for the linear term of the linear model was calculated in all models with the smallest error, while the estimated coefficients of the nonlinear model were characterized by much larger errors.

\section{CONCLUSION}

The conducted research allows for the following conclusions:

- The choice of basic functions approximating the measuring instrument processing function is very important from the point of view of the accuracy of determining the model estimators.

- The nonlinearity of the model of the measuring instrument processing function can significantly increase the determinant factor of the UTU matrix of the system of equations. As a result, the calculated estimators show high sensitivity to errors in the measurement data contained in the $Z$ vector.

- The systematic error of the measurement, simulated by the noise mean value, has a significant influence on the residual sum of squares RSS, which is related to the accuracy of the approximation.

- Bearing in mind the above statements, the calibration of a measuring instrument should take into account, i.e., the influence of the approximation model of the processing function on the assessment of the measuring instrument capability.

\section{REFERENCES}

Arendarski, J., Gliwa-Gliwińśki, J., Jabłoński, Z., Ratajczyk, E., Tomasik (red.), J., Żebrowska-Łucyk, S., 2009. Sprawdzanie przyrządów do pomiarów długości i kąta, Oficyna Wydawnicza Politechniki Warszawskiej, Warszawa.

Bellman, R., 1960. Introduction to matrix analysis, McGraw-Hill, New York.

Doyle, J., F., 2004. Modern experimental stress analysis, Willey, Chichester.

Draper, N.R., Smith, H., 1981. Applied regression analysis, Willey, New York.

Hagel, R., 1975. Miernictwo dynamiczne, Wydawnictwa Naukowo-Techniczne, Warszawa.

Horn, R.A., Johnson, C., 1986. Matrix analysis, Cambridge University Press, Cambridge.

IEC 60050-301:2000. International Electrotechnical Vocabulary (IEV) - Part 301: General terms on measurements in electricity.

International Dictionary of Basic and General Metrology Terms, 1993. Central Office of Measures, Warsaw.

ISO 14978:2018. Geometrical product specifications (GPS) - General concepts and requirements for GPS measuring equipment

ISO 9000:2015a. Quality management system - Fundamentals and vocabulary. 
ISO 9001:2015b. Quality management systems - Requirements.

ISO/IEC 17025:2017. General requirements for the competence of testing and calibration laboratories

ISO/IEC Guide 99:2010. International vocabulary of metrology - Basic and general concepts and associated terms (VIM)

Jakubiec, W., Malinowski, J., 2004. Metrologia wielkości geometrycznych, Wydawnictwa Naukowo-Techniczne, Warszawa.

Jakubiec, W., Zator, S., Majda, P., 2014. Metrologia, Polskie Wydawnictwo Ekonomiczne S.A., Warszawa.

Lawson, C., Hanson, R.J., 1974. Solving least squares problems, Prentice-Hall, Englewod Cliffs.

Ostasz G., Czerwińska, K., Pacana, A., 2020. Quality management of aluminum pistons with the use of quality control points, Management Systems in Production Engineering, 28(1), 29-33.

Pacana A., Czerwińska K., Bednarowa L., 2018. Comprehensive improvement of the surface quality of the diesel engine piston, Metalirgija, 58, 3-4, 2018, 329-332.

Piotrowski, J., Kostyrko, K., 2012. Wzorcowanie aparatury pomiarowej, Wydawnictwo Naukowe PWN S.A., Warszawa.

Siwiec, D., Bednárowá, L., Pacana, A., Zawada, M., Rusko, M., 2019. Decision support in the selection of fluorescent penetrants for industrial non-destructive testing. Przemysł Chemiczny, 98(10), 1594-1596.

Siwiec, D., Pacana, A., 2021. Method of improve the level of product quality.

Production Engineering Archives, 27(1), 1-7.

https://doi.org/10.30657/pea.2021.27.1

Tikhonov, A.N., Arsenin, V.Y., 1977. Solutions of ill-posed problems, Willey, New York. Willkinson, J.H., 1965. The algebraic eigenvalue problem, Clarendon Press, Oxford. 